\title{
激光直写制造石墨烯基柔性电子器件的研究进展
}

\author{
陆龙生 ${ }^{1,2}$ 王文涛 ${ }^{1,2}$ 谢颖熙 ${ }^{1,2}$ 汤 勇 ${ }^{1,2}$
}

(1. 华南理工大学机械与汽车工程学院 广州 510640;

2. 华南理工大学广东省功能结构与器件智能制造工程实验室 广州 510640)

\begin{abstract}
摘要: 柔性电子器件相对于传统电子器件, 拥有独特的柔性和延展性, 能够在一定程度上适应不同的工作环境, 满足设备的 形变需求。石墨烯是开发柔性电子器件的理想材料。然而, 传统的石墨烯加工技术大多涉及高温和化学溶剂, 存在着成本高, 工艺线路复杂和环境污染等问题, 并不适合未来产业发展。激光直写(Laser direct writing, LDW)技术具有加工速度快, 扫描面 积大和空间分辨率高等优点, 且无需掩模和后处理, 在现代工业中广泛应用。最新研究表明, 激光直写技术可以从氧化石墨 烯、多种聚合物甚至天然材料中衍生出石墨烯, 这无疑进一步提升了石墨烯基柔性电子器件的应用潜力。本文对现有激光直 写技术制备石墨烯的前驱体进行了归纳分类, 并详细介绍了相应的演变过程、加工原理及辅助加工设备, 总结了基底转印、 表面应变结构、剪纸拓扑结构这三种常见的柔性化制造策略, 并重点阐述了其在超级电容器、传感器、纳米发电机和致动器 等石墨烯基柔性电子器件中的最新应用, 最后对其发展趋势及挑战进行讨论。
\end{abstract}

关键词：激光直写；石墨烯；柔性电子器件；制造方法

中图分类号: TH165

\section{Recent Advances in the Fabrication of Graphene-based Flexible Electronic Devices by Laser Direct Writing}

\author{
LU Longsheng ${ }^{1,2}$ WANG Wentao ${ }^{1,2}$ XIE Yingxi ${ }^{1,2}$ TANG Yong ${ }^{1,2}$ \\ (1. School of Mechanical and Automotive Engineering, South China University of Technology, Guangzhou \\ 510640 ;
}

2. Intelligent Manufacturing Engineering Laboratory of Functional Structure and Devices in Guangdong Province, South China University of Technology, Guangzhou 510640)

\begin{abstract}
Compared with traditional electronic devices, flexible electronic devices have unique flexibility and ductility, which can adapt to different working condition and meet the deformation needs of equipment. Graphene is an ideal material for developing flexible electronic devices. However, traditional graphene processing technologies often involve high temperature and chemical solvents, require high-cost, complicated technical routes and environmental pollution, which are not suitable for future development. Laser direct writing (LDW) technology does not require masks and post-processing, and has the advantages of fast processing speed, large scanning area, and high spatial resolution. Therefore, it has been widely used in modern industry. Recent research showed that LDW can produce graphene from a variety of polymers and even natural materials, which undoubtedly further enhances the potential of graphene in flexible electronic devices. In this work, the precursors of graphene prepared by LDW are summarized, and the corresponding evolution process, processing principle and auxiliary processing equipment are introduced in detail. The huge advances in the design and manufacture of flexible electronic devices by the three strategies of transfer process, strain-accommodating engineering and Kirigami-inspired structure, and their latest applications in supercapacitors, sensors, nanogenerators and actuators are summarized. Furthermore, the development trend and challenges are discussed.
\end{abstract}

Key words: laser direct writing; graphene; flexible electronic devices; manufacturing method

* 国家自然科学基金资助项目(51775197)。20201221 收到初稿, 20210318 收到修改稿 


\section{0 前言}

在数十万年的历史长河中, 科技在不断地发展, 人类从原始的 “石器时代” 来到了充满科技感的 “信 息时代”。当前, 电子信息产业沿着硅基的道路发展, 仍然以刚性器件为主 ${ }^{[1]}$ 。然而, 受摩尔定律的影响, 经典硅基电子学的 “瓶颈” 已日益凸显 ${ }^{[2]}$ 。事实上, 早在 1974 年, 一种名为 “Gyricon” 的柔性电子纸 的诞生, 就已经给人们指明了方向。它不仅可以显 示图像信息, 而且颠覆了传统电子器件的刚性特征, 能够像纸一样弯曲, 以达到功能、信息与人类活动 的高效互融。通常, 柔性电子器件以柔性材料为基 底, 结合微纳米加工与制造技术, 可以实现能源存 储/收集、信号传感等功能, 大有 “以柔克刚” 的意 味 ${ }^{[3]}$ 。然而, 柔性电子器件的大规模商业化仍面临 着两大挑战。首先, 柔性电子器件需要在不损坏本 身电子性能的基础上, 保留一定机械柔性, 特别是 可拉伸性, 以适应不同的工作环境; 其次, 组成电 路的各种柔性电子器件也在逐渐向多功能化和微型 化进行发展, 开发相应的制造技术和性能均衡的原 材料十分必要 ${ }^{[4]}$ 。

石墨烯是一种由碳原子以 $\mathrm{sp}^{2}$ 杂化轨道组成的六 角型蜂巢结构的二维碳纳米材料 ${ }^{[5]}$ 。这种独特而奇妙 的结构赋予了其空前的优越性能, 包括: 高电荷载流 子迁移率 $\left(200000 \mathrm{~cm}^{2} \cdot \mathrm{V}^{-1} \cdot \mathrm{s}^{-1}\right)$ 、出色的光学透射率 $(\sim 97.3 \%)$ 、室温下的优异导热系数(3000 $\left.5000 \mathrm{~W} \cdot \mathrm{m}^{-1} \cdot \mathrm{K}^{-1}\right)$ 、理论上的高表面积 $\left(\sim 2600 \mathrm{~m}^{2} \cdot \mathrm{g}^{-1}\right)$ 和高弹性模量 $(\sim 1.0 \mathrm{TPa})$ 等 $^{[6]}$ 。因此, 石墨烯及其衍生 物已广泛用于制造各种柔性电子器件。目前, 石墨烯 制备方法主要有机械剥离、化学气相沉积、溶剂分
解和化学合成等。但是, 这些方法都涉及高温和化 学溶剂, 存在着成本高, 技术线路复杂和环境污染 等问题, 并不适合于实际生产 ${ }^{[7]}$ 。并且, 与石墨烯 的制备相比, 石墨烯的加工方法, 特别是用于柔性 电子器件的制造技术仍处于早期阶段。

激光直写 (Laser direct writing, LDW)技术作为 一种无需掩模和后处理的制造方法, 以其处理速度 快、扫描面积大和空间分辨率高等优点受到了广泛 的关注 ${ }^{[8]}$ 。最初, 激光直写技术主要通过烧蚀、蚀 刻、切割和诱导等方式, 直接在各种材料上进行表 面处理, 或对各种集成器件进行修饰, 以实现功能 化 ${ }^{[9]}$ 。最新研究表明, 激光直写技术不仅与现有电 子工业的各种集成设计技术相容, 而且能够从氧化 石墨烯、多种聚合物甚至天然材料中衍生出石墨烯, 引起了国内外学者的广泛关注 ${ }^{[10]}$ 。如表 1 所示, 虽 然传统的石墨烯基柔性电子器件制造工艺, 例如: 光刻、等离子刻蚀、丝网印刷和喷墨打印等, 已经 较为成熟, 但其性能往往受到石墨烯重新堆积和团 聚现象的限制, 表现较不理想 ${ }^{[7,11]}$ 。相反, 基于激光 直写技术制造的石墨烯基柔性电子产品不会过分依 赖于硅基半导体技术，具有独特的可拓展性以及高 效、低成本的制造工艺，非常适合当前工业的大规 模生产 ${ }^{[12-18]}$ 。

本文针对激光直写技术制备石墨烯基柔性电 子器件这一研究热点, 从激光加工的角度出发, 详细介绍了激光直写制备石墨烯的前驱体类型、 加工条件和辅助加工设备等, 并结合电子器件常 用的柔性化制造方法, 阐述了其在超级电容器、 传感器、纳米发电机和致动器等诸多柔性电子器 件的应用进展和存在的问题, 并对其进行了科学 预测与展望。

表 1 石墨烯基柔性电子器件的常规制造方法

\begin{tabular}{|c|c|c|c|c|}
\hline 方法 & 核心工艺 & 优点 & 缺点 & 文献 \\
\hline 光刻法 & 涂胶、曝光、显影、烘干 & 低成本、任意基底 & $\begin{array}{l}\text { 光致抗蚀剂污染、多步 } \\
\text { 骤、多缺陷 }\end{array}$ & {$[12]$} \\
\hline 等离子刻蚀法 & 旋涂、热退火、曝光 & 低成本、大面积 & 高温、低效率、多步骤 & {$[13]$} \\
\hline 化学气相沉积法 & 通气、催化、反应 & 大面积、高质量 & 限 高温、高成本、产物有 & {$[14]$} \\
\hline 丝网印刷 & 制板、印刷、烘干 & 简便、低成本、应用范围广 & 耐久性差、精度低 & {$[15]$} \\
\hline 喷墨打印法 & 建模、打印、后处理 & 设计空间大、多样化 & $\begin{array}{l}\text { 材料限制、需要载体、 } \\
\text { 质量和精度低 }\end{array}$ & {$[16]$} \\
\hline 激光直写法 & $\begin{array}{l}\text { 各种气氛条件下 }\left(\text { 空气、 } \mathrm{O}_{2} 、 \mathrm{~N}_{2} 、\right. \\
\mathrm{Ar} \text { )直写 }\end{array}$ & $\begin{array}{l}\text { 大面积、快速、高精度、非 } \\
\text { 接触、无需掩模、前驱体多 }\end{array}$ & $\begin{array}{c}- \\
-\end{array}$ & {$[17,18]$} \\
\hline
\end{tabular}

\section{1 激光直写制备石墨烯的研究现状}

通常, 前驱体的化学组成和物理特性决定了石
墨烯的基本性能, 进一步影响其在柔性电子器件中 的应用前景 ${ }^{[19]}$ 。目前, 能够通过激光直写技术制备 石墨烯的前驱体数量繁多, 主要可分为氧化石墨烯、 聚合物和天然材料三大类。另外, 从电子器件柔性 
化制造的角度来看, 本身具有柔性特征的聚合物可 以同时充当基底和前驱体, 更具有实际研究价值, 而以低成本、来源丰富和无污染的天然材料作为基 底进行绿色电子产品的加工制造, 将成为未来的研 究热点。

\section{1 氧化石墨烯}

氧化石墨烯(Graphene oxide, GO) 是一种常见的 石墨烯衍生物, 由于碳纳米片上存在许多含氧基团 而处于绝缘状态。所以, 通过激光直写技术将 $\mathrm{GO}$ 还原为石墨烯遵循两个基本过程: (1) 高温伴随着化 学反应, 从 GO 表面去除含氧基图案; (2) 破坏的化 学键之间进行重组, 形成 $\mathrm{sp}^{2}$ 共轭石墨烯平面六边 形结构 ${ }^{[20]}$ 。

事实上, 激光直写 GO 制备石墨烯的本质是光 还原过程, 而根据 $\mathrm{GO}$ 吸收的光子能量不同, 又可 分为光热还原过程和光化学还原过程。2011 年, SMIRNOV 等 ${ }^{[21]}$ 报道的阈值理论指出, 超过 $3.2 \mathrm{eV}$ 的光子能引发光化学还原, 而小于此阈值的光子能 引发光热还原。因为 $\mathrm{GO}$ 具有从紫外到近红外区域 的宽光吸收带, 所以各种光源, 甚至是阳光, 都可 以触发有效的光还原反应。与常规的热/化学手段相 比, 光还原不仅可以有效减少 $\mathrm{GO}$ 的含氧基团, 制 备出高质量的石墨烯, 而且会因为水分子和碳物质 在加工过程中的大量释放而产生疏松多孔结构。 2010 年, 吉林大学孙洪波课题组 ${ }^{[22]}$ 采用紧密聚焦的 飞秒激光束在 GO 膜上进行还原, 加工出了石墨烯 微电路。结果表明, 通过改变激光功率, 不含氧基 团的碳含量从 $38 \%$ 增加至 $83 \%$, 为激光直写制备石 墨烯及其微结构化开辟了一条新的途径。

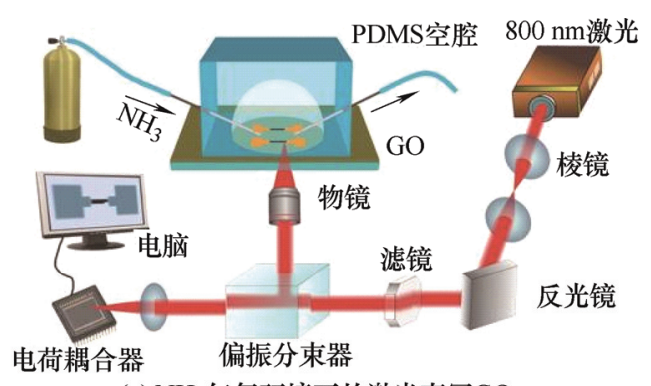

(a) $\mathrm{NH}_{3}$ 气氛环境下的激光直写 $\mathrm{GO}$

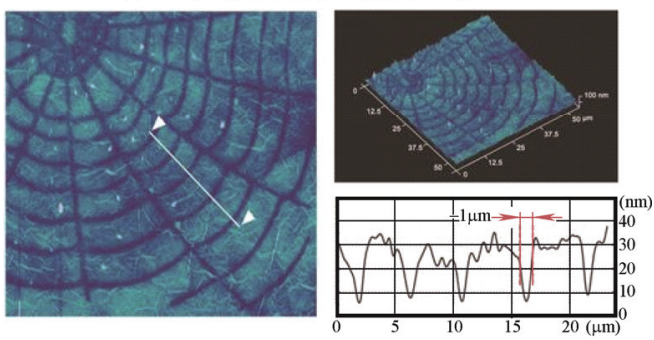

(b) 激光直写 $\mathrm{GO}$ 的图案化及表面形貌

图 1 激光直写 $\mathrm{GO}$ 的 $\mathrm{N}$ 掺杂和蜘蛛网图案的构建 ${ }^{[24]}$
后来, 关于激光直写 $\mathrm{GO}$ 的无掩模图案化, 分 层结构化、局部杂原子掺杂和金属/金属氧化物复合 等研究也逐步展开 ${ }^{[23]}$ 。大家意识到, 尽管热/化学方 法已经广泛用于石墨烯的生产，但其与柔性电子器 件的加工设备和制造工艺往往不兼容, 尤其是在形 状极不固定的柔性基板上进行。相比之下, 通过 GO 的激光直写来制备石墨烯, 特别是在特殊环境 $\left(\mathrm{NH}_{3} 、 \mathrm{~N}_{2} 、 \mathrm{Ar}\right.$ 等气体和水、乙醇等液体)下进行加 工, 可实现石墨烯的高精度图案化。如图 1 所示, GUO 等 ${ }^{[24]}$ 通过飞秒激光在 $\mathrm{NH}_{3}$ 气氛中直写 $\mathrm{GO}$ 并 进行 $\mathrm{N}$ 掺杂, 实现了大约 $500 \mathrm{~nm}$ 分辨率的石墨烯 蜘蛛网图案的制作, 其粗粘度和表面形貌高度可控。

如图 2a 所示, ABDELSAYED 等 ${ }^{[25]}$ 发现无需添 加任何化学还原剂, 可以在水溶液中激光还原的单 个石墨烯片。GO 的透过率和电阻率在不同波长激 光照射下呈现不同的变化规律, 其中, 波长为 $532 \mathrm{~nm}$ 和 $355 \mathrm{~nm}$ 激光都能将溶液中的 $\mathrm{GO}$ 有效地 转化为石墨烯, 但使用功率相近的 $1064 \mathrm{~nm}$ 激光器, 由于 $\mathrm{GO}$ 的光吸收率较低而无法还原。如图 $2 b$ 所示, HUANG 等 ${ }^{[26]}$ 通过 $248 \mathrm{~nm} \mathrm{KrF}$ 准分子激光直写溶液 中的 GO, 黄棕色的 GO 溶液在几分钟内变黑, 通 过激光还原除去大多数含氧基团之后, 石墨烯薄片 的厚度几乎与原来的 $\mathrm{GO}$ 相同, 都为 $1.1 \mathrm{~nm}$ 左右。

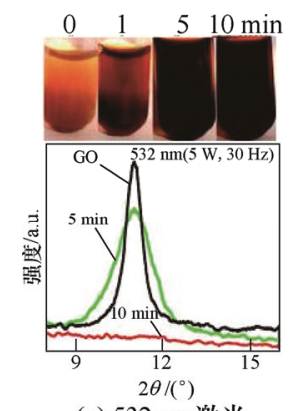

(a) 532 nm激光

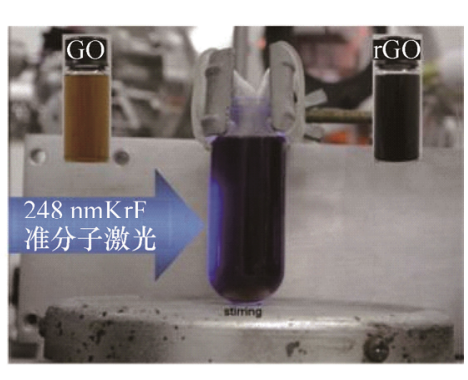

(b) $248 \mathrm{~nm} \mathrm{KrF}$ 准分子激光
图 2 不同类型激光器在溶液中还原 $\mathrm{GO}^{[25-26]}$

\section{2 聚合物}

虽然激光直写技术可以在 $\mathrm{GO}$ 片层、薄膜和溶 液中高效制备石墨烯并图案化, 但 GO 生产过程需 要到大量酸性和氧化性化工原料, 涉及不可逆的生 产过程, 且成本依然过高, 所以许多学者开始寻找 GO 的替代品。早在 20 世纪 90 年代初期, SRINIVASAN 等 ${ }^{[27]}$ 就报道了关于激光直写聚酰亚 胺(PI)薄膜原位热解和碳化的研究, 可在当时被认为 是一种简单的烧蚀行为, 没有引起足够的重视。

2014 年, 莱斯大学的 TOUR 的课题组 ${ }^{[28]}$ 在研究 高功率 $\mathrm{CO}_{2}$ 激光在空气中直写 PI 薄膜时, 发现这种 商业化产品可以原位转化为松散堆积的多孔石墨 
烯, 并将其命名为激光诱导石墨烯(Laser-induced graphene, LIG)。随后, 该课题组继续通过激光直写 多达 15 种聚合物后发现, 只有包含芳族和酰亚胺重 复单元的 PI 和聚醚酰亚胺(PEI)可以还原为 $\mathrm{LIG}$ 。之 后, 其他课题组也陆续报道了一些聚合物, 如磺化 聚醚醚酮、聚砜、聚醚砜、酚醛树脂等也适合 LIG 的生产 ${ }^{[29]}$ 。相比于直接加热的方式碳化聚合物, 激 光直写技术具有高效、图案化和高度可控的特点, 并且能够大规模生产应用。

除了在材料方面进行突破之外, 不少加工设备 也相继研发出来, 用于激光直写石墨烯的扩展性研 究。2017 年, LI 等 ${ }^{[30]}$ 设计了一个封闭的腔室激光直 写 PI 薄膜, 通过加入不同气氛 $\left(\mathrm{Ar} 、 \mathrm{O}_{2}\right.$ 和 $\left.\mathrm{H}_{2}\right)$, 可 以控制 LIG 的原子百分比形貌结构, 从而有效地调 节了表面润湿性。2018 年, LUONG 等 ${ }^{[31]}$ 开发了一 种直接生产三维 LIG 的层压件的制造方法, 如图 3 所示。首先, 将两个表面带有 LIG 的 PI 片材通过乙 二醇粘接在一起, 再使用 $10.6 \mu \mathrm{m}$ 激光将裸露的 PI 面转换为 $\mathrm{LIG}$, 最后通过重复构建三明治结构和激 光直写, 制备了 $3 \mathrm{D}$ 的 $\mathrm{LIG}$ 层压件。由于 $\mathrm{CO}_{2}$ 激光 的波长限制, 产物的边缘分辨率较差。基于此, 使 用 $L I G$ 可吸收但对 PI 透明的 $1.06 \mu \mathrm{m}$ 的光纤激光器 对产物结构进行铣削来控制尺寸特征。这种方法与 3D 打印方法类似, 可以制造多维度的 LIG 基柔性 电子器件。

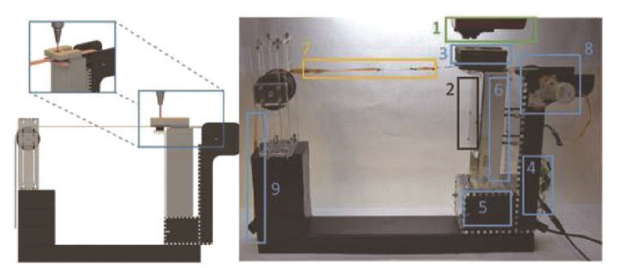

(a) 激光直写PI薄膜制备3D石墨烯层压件

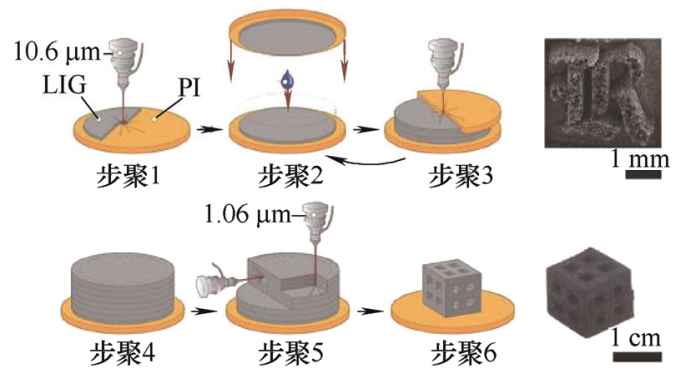

(b) 激光直写及铣削工艺

图 3 激光直写 PI 薄膜制备 $3 \mathrm{D}$ 石墨烯层压件 ${ }^{[31]}$

激光直写石墨烯的产业化对于未来工业发展十 分重要。如图 4 所示, WANG 等 ${ }^{[32]}$ 报道了一种通过 $10.6 \mu \mathrm{m}$ 的 $\mathrm{CO}_{2}$ 激光逐行直写 PI 纸来生产石墨烯的 工艺方法。由于尺寸和参数设置的限制, 在实验室 中, 石墨烯的生产效率可以达到大约 $30 \mathrm{~cm}^{2} \cdot \mathrm{min}^{-1}$,
最大生产尺寸接近 $1400 \mathrm{~cm}^{2}$ 。另外, 纸质独特空间 结构使得本身具有良好的稳定性, 不会出现大变形。 在大功率激光器的辅助下, 纸质石墨烯可以切割和 修整成任意形状, 具有低成本, 快速, 任意尺寸, 易于操作的特点。这种实验室规模的生产方式有望 进一步升级为卷对卷的连续制造, 以实现石墨烯的 大规模生产。

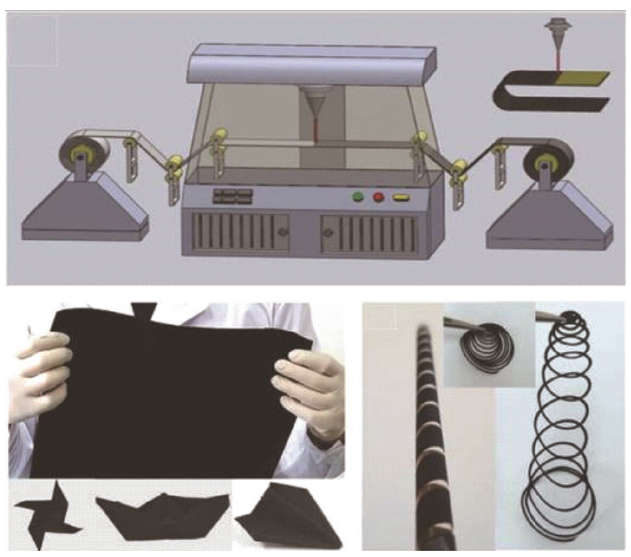

图 4 激光直写制造石墨烯纸的卷对卷工艺及实物图 ${ }^{[32]}$

\section{3 天然材料}

据报道, 2020 年后, 中国每年或产生 1 亿吨电 子垃圾, 其材料大多来源于石油、煤炭等不可再生 资源, 耗费巨大, 且对人类生存环境影响恶劣。考 虑到聚合物为前驱体的成本仍然较高, 且大多数不 能生物降解, 开发来源丰富且绿色环保的天然材料 作为激光直写制备石墨烯的前驱体, 并进一步制造 柔性电子器件具有重要的意义。

2017 年, TOUR 的课题组 ${ }^{[33]}$ 使用 $\mathrm{CO}_{2}$ 激光在惰 性气氛下对天然木材进行直写生产石墨烯。通过研 究不同类型的木材, 包括: 松木, 桦木和橡木等发 现，芳香木质素含量和木材固有的复合结构是影响 LIG 质量的关键因素。2018 年, YIEU 等 ${ }^{[34]}$ 进一步 改善了激光直写工艺, 开发了一种利用 $\mathrm{CO}_{2}$ 激光将 大多数天然含碳材料, 例如: 椰壳、纸板、纱布和 面包等转化为 LIG 的方法。如图 5 所示, 首先使用 阻燃剂或炭化对基材进行预处理, 然后在祄底上进 行多次激光处理, 或采用激光散焦多次加工。研究 表明, 在激光直写过程中, 基材首先转变为非晶碳, 进一步诱导转变为 LIG, 具有很高的导电性, 薄层 电阻低至 $\sim 5 \Omega \cdot \mathrm{sq}^{-1}$ 。

2019 年, LE 等 ${ }^{[35]}$ 利用飞秒激光的超快光脉冲 特性, 在天然木材和树叶上一步直写形成高导电 LIG 图案, 并充分研究了木材到石墨烯的转变过程 及机理。如图 6 所示, 超短脉冲持续时间和低脉冲 通量的激光加工参数可以产生大量的热并减少了热 
扩散对材料的负面影响, 有效地将木材细胞转换为 LIG 而不会造成显著的烧蚀或热损伤。另外, 木材和 树叶的所有成分, 包括: 含有酚基的木质素、由糖单 体组成的纤维素和半纤维素等都可以转化成 $L I G$ 。这 项研究为生物相容、生物可降解和生态友好的石墨烯 基绿色电子产品的大规模生产提供了蓝图。

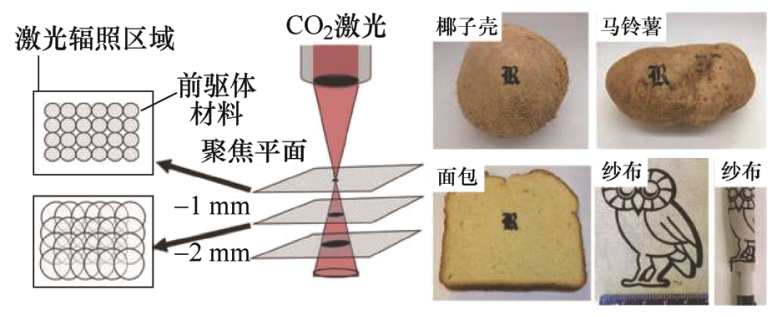

图 5 激光直写多种天然含碳材料制备石墨烯[34]

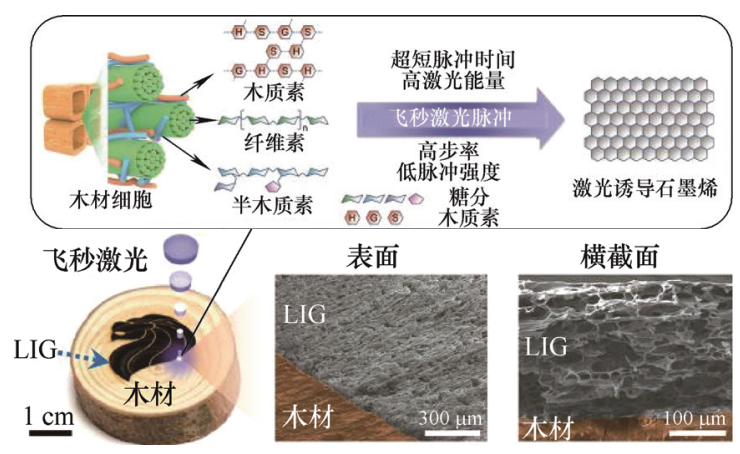

图 6 激光直写木材生成石墨烯的工艺过程及形貌 ${ }^{[35]}$

多环重烃, 例如煤, 焦油和沥青等是一类化学 成分极其丰富和复杂的天然材料, 也是现代工业生 产中不可或缺的原料。2020 年, 麻省理工学院的 ZANG 等 ${ }^{[36]}$ 在一系列多环重烃上进行激光直写制备 了不同质量的石墨烯。如图 7 所示, 初始原料化学 成分 (原子比和芳烃含量) 与激光处理参数(激光功 率、扫描速度和焦距)与最终石墨烯的 $\mathrm{C}$ 含量, $\mathrm{sp} 2$ 浓度和石墨堆叠顺序息息相关。与此同时, 碳材料 的结晶度涉及无定形到高度石墨化的广泛分布, 以 及高达 $103 \mathrm{~S} \cdot \mathrm{m}^{-1}$ 的电导率, 是激光直写技术制备高 度可调的石墨烯的关键, 也为其在电子信息、化工 机械、生物医疗等一系列潜在领域的应用提供了技 术支持。

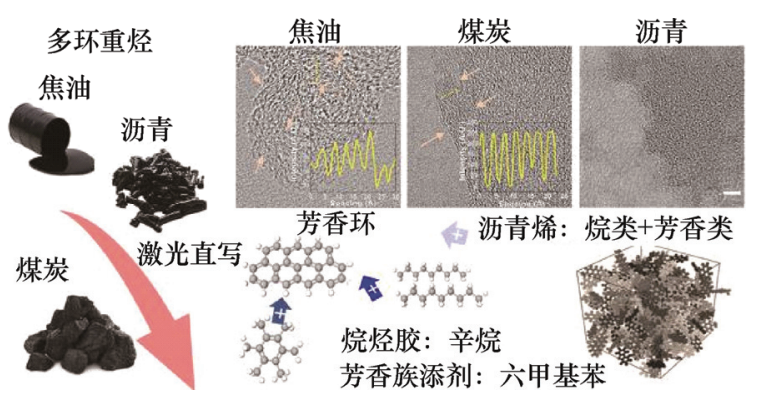

图 7 激光直写多环重烃生成石墨烯的微观形貌及原理 ${ }^{[36]}$

\section{2 激光直写制备石墨烯的柔性化制造}

在日常使用中, 电子器件可能会遇到一些不可 避免的机械变形, 所以柔性化成为未来电子器件发 展的必然趋势。柔性电子器件制造的关键在于电极/ 电路的柔性化, 这直接决定了其在任意形变条件下 (例如: 弯曲、折叠和拉伸等)保持稳定性能的能力。 目前, 大多数柔性电子器件的研究主要集中在弯曲 形变条件下的电子性能, 而拉伸是另一种常见的机 械变形, 由于前驱体和石墨烯本身的刚性限制, 难 以实现却颇具现实意义。因此, 国内外学者纷纷开 展了对石墨烯基电子器件的柔性化设计和制造理论 研究, 主要包括: 柔性基底转印法、表面应变结构 设计法和剪纸拓扑结构法等。

\section{1 柔性基底转印法}

在激光直写制备石墨烯的同时, 可以进行电极 的图案化, 但考虑到大多数前驱体都是刚性的, 无 法满足电子器件的柔性化需求。于是, 一种将图案 化的石墨烯电极结构有序地转移到另一种弹性基底 上的方法, 即转印法, 已被广泛应用于制造各种柔 性电子器件。与其他柔性化制造技术相比, 转印法 具有以下优点: (1) 在常温下进行, 避免了温度可能 对器件功能带来的影响; (2) 技术兼容性强, 可以与 很多其他加工技术相兼容; (3) 操作简单, 无论是纳 米线、二维结构, 还是复杂的三维多层结构, 都可 以利用转印技术进行转移和组装。

弹性基体是柔性电子产品拥有形变能力的基 础, 其必须具备优异的拉伸性能和可回复性, 同时 化学稳定性高, 能够适应各种恶劣环境并稳定工作。 聚二甲基硅氧烷(PDMS)是目前转印最常用的弹性 基体, 制备工艺十分简单。如图 8 所示, LAMBERTI 等 ${ }^{[37}$ 通过纳秒级 $\mathrm{CO}_{2}$ 激光直写 PI 薄膜生成多孔的 LIG 图案, 然后将 PDMS 倒入图案上, 并通过真空 步骤将空气抽空, 使 PDMS 完全渗透到石墨烯多孔 结构中, 在 $80{ }^{\circ} \mathrm{C}$ 下热固化 $1 \mathrm{~h}$ 后, 即可手动从 PI 膜上剥离出具有高拉伸性的透明 LIG/PDMS 电极, 该材料可拉伸至 $50 \%$ 或弯曲 $160^{\circ}$ 。

橡胶/乳胶是一种高度可逆形变的弹性聚合物 材料, 具有优异的拉伸性能和快速的回复能力。 SONG 等 ${ }^{[38]}$ 在 PI 薄膜上激光直写制备了 $3 \mathrm{D}$ 多孔网 络结构的石墨烯, 然后采用转印法将其转移到硅橡 胶基板上以获得高度可拉伸且透明的 LIG 电极。相 比于 LIG/PDMS 电极, 该 $\mathrm{LIG}$ /硅胶电极最高可拉伸 至 $400 \%$ ，且可靠性和耐疲劳大幅度提高。 


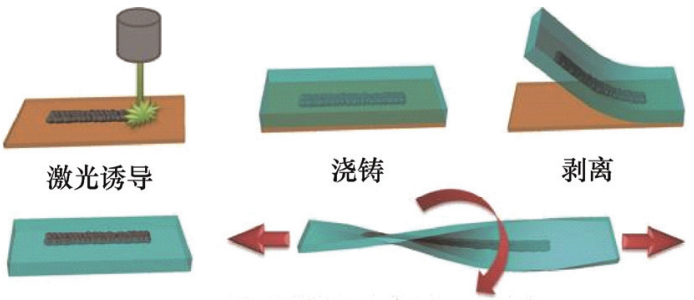

(a) 柔性基底转印的主要工艺流程

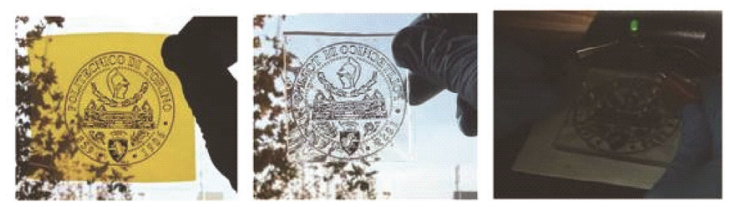

(b) 转印后的柔性LIG/PDMS石墨烯图案

图 8 柔性基底转印流程及 LIG/PDMS 石墨烯图案 ${ }^{[37]}$

事实上, 通过转印法所制备的石墨烯电极可以 满足多种形变需求, 但所采用的大多数基体都不能 在自然条件下降解, 对人类生活环境构成潜在威胁。 聚氨酯是一种新型的高分子材料, 也是目前广泛使 用的弹性基体，属于常见的生物可降解性材料。同 时, 一些水性聚氨酯也可以通过转印法用来制备柔 性电极。WANG 等 ${ }^{[39]}$ 通过可降解的水性聚氨酯来转 印激光直写 PI 薄膜所生成的石墨烯电极, 并充分研 究了转印前后的形貌演变和拉伸过程中的石墨烯结 构变化。结果发现, 可拉伸的聚氨酯微纤维在拉伸/ 松驰过程中可以连接相邻石墨烯碎块, 使得整体的 可拉伸性能和导电性能得到了充分的提高。但是, 聚氨酯的耐水较差, 而且对酸碱也较为敏感, 这是 在实际使用中所要考虑的问题。

除了单一的利用基体转印之外, SUN 等 ${ }^{[40]}$ 报道 了一种简单有效的方法来制作具有高透气性的多孔 柔性石墨烯电极, 具体以疏松多孔的 LIG 为电极, 然后在硅弹性体中加入食糖作为衬底, 待水溶解糖 分之后, 制备类似海绵体为柔性祄底。这不仅丰富 了柔性基体转移法制备柔性电子器件的手段, 还提 高了器件性能, 扩大了应用范围。

上述所使用的方法, 都需要分两步进行, 即 激光直写石墨烯和目标基底的浇铸和剥离, 操作 较为复杂, 不适合实际生产。 $\mathrm{OH}$ 等 ${ }^{[41]}$ 开发了一 种使用连续波二极管激光将 GO 从基材直接图案 化并转移到柔性塑料薄膜上的方法, 其加工机理 和制造过程如图 9 所示。首先, 激光直写 GO 薄 膜使得聚对苯二甲酸乙二醇酯(PET)薄膜的局部 熔化, 然后, 在冷却时对石墨烯-PET 界面进行牢 固焊接, 激光产生的热量就可以将 GO 转换为具 有导电性的石墨烯图案, 并且转印后 PET 上的石 墨烯图案非常清晰。

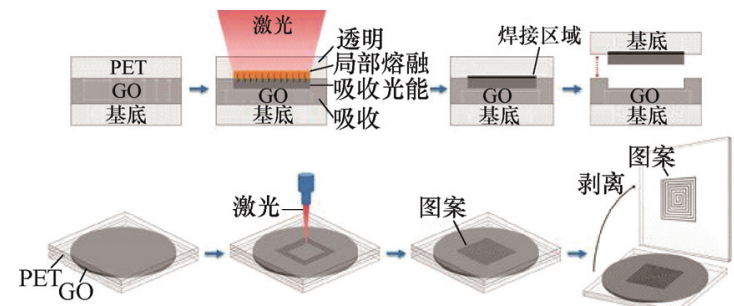

(a) 激光直写/转印石墨烯制备柔性电极工艺图

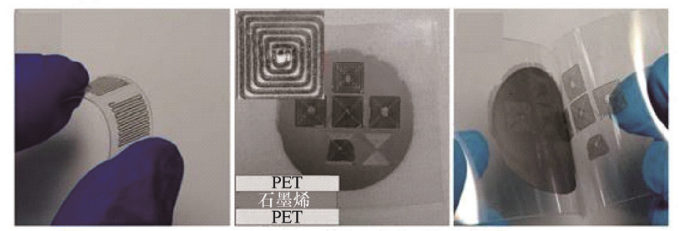

(b) 柔性电极图案的实物展示图

图 9 一步法实现激光直写 $\mathrm{GO}$ 的图案化和转印工艺 ${ }^{[41]}$

\section{2 表面应变结构设计法}

表面应变结构设计法主要通过非线性屈曲或平 面内弯曲过程来响应应变, 赋予电子器件极大的柔 性。同时, 可以提供电子器件相应的有效拉伸, 避 免电极材料在正常工作中发生显著变形。目前, 常 见的结构设计种类较多, 如图 10 所示, 根据形状特 点, 大致可分为波浪状、带状、褶留状、蛇形状、 线圈状和网状等 ${ }^{[42]}$ 。事实上, 这些设计可以适用于 多种材料而不考虑其内在特性和尺寸，甚至构成传 统电子设备基础的脆性无机材料, 都可以根据几何 布局进行柔性化设计和制造。
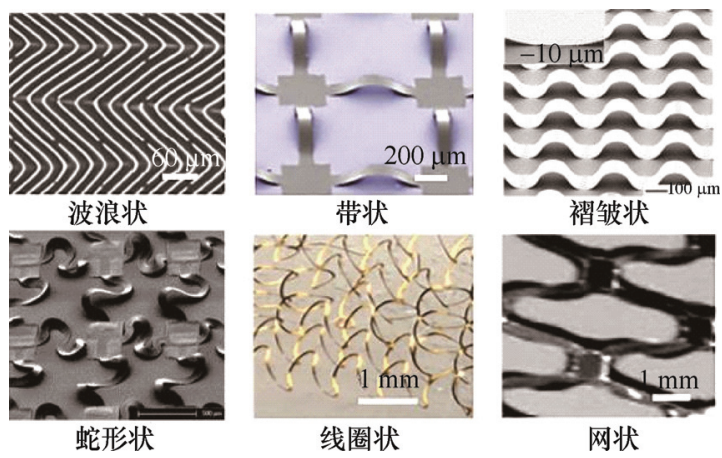

图 10 常见的表面应变结构设计 ${ }^{[42]}$

褶皱结构, 也可以看作三维立体的波浪状结构, 具有工艺简单、可靠性好、可拉伸性强等优点, 在 柔性电子器件的结构设计中得到了广泛的应用。一 般来说, 褶皱结构可以通过“预应变-传递-释放”方法 来实现柔性化, 通常包括三个步骤: (1) 在弹性基底 进行多维度预应变设计; (2) 将活性电极材料转移至 预应变基底表面; (3) 有序且不同程度的释放弹性基 底中预应变。该方法的设计关键在于内部所存储的 扩展空间, 它可以释放拉伸应力, 减少拉伸应力对 导电材料的破坏，从而保持稳定的导电性。

通常, 电极材料是通过旋涂、原位诱导和电化 
学沉积等多种工艺贴附在弹性基体的应变结构表 面, 起到主体功能作用。因此, 导电材料和弹性基 体之间的表面结合力至关重要, 吸附能力不足容易 导致器件性能下降甚至失效。基于此, QI 等 ${ }^{[43]}$ 通过 将石墨烯微带转移到具有三脚架微结构的 PDMS 基 板上, 合理地设计了高度可拉伸的悬浮波浪状石墨 烯电极阵列。如图 11 所示, 与大多数电极材料和基 底间接触的方式不同, 中空悬浮的波浪状结构将大 幅减少拉伸/松驰过程中电极的应变集中; 同时, 确 保叉指电极在拉伸/松弛过程中保持相对恒定的距 离, 提高了柔性电子器件的稳定性。另外, 通过有 限元建模分析可以得出不同电极阵列及其相关应变 分布, 以便对实际应用中可能遇到的形变进行预测。

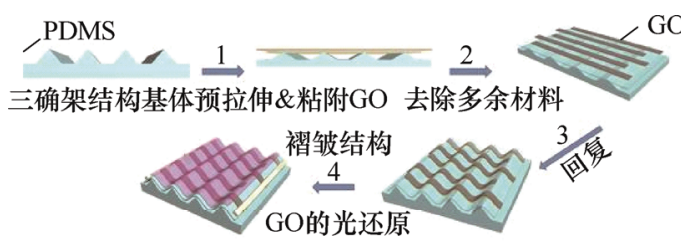

(a) 微结构基体预拉伸制备 $\mathrm{rGO}$ 波浪结构

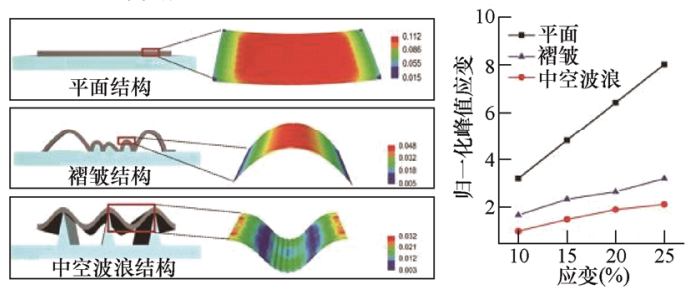

(b) 三种结构的有限元分析及应力应变分析

图 11 悬浮波浪状石墨烯电极结构设计及有限元分析 ${ }^{[43]}$

\section{3 剪纸拓扑结构设计法}

据记载, 早在春秋战国时期, 人们就通过镂空 雕刻的技法在一些薄片材料上制作工艺品。后来, 这种使用剪刀或刻刀在纸上剪刻花纹的图案手法逐 渐成了一种古老的民间艺术, 在窗花、剪影、皮影 戏等多种中国传统元素中均有体现。同时, 科学家 们以此为启发, 创造性地将剪纸拓扑结构作为一种 独特的力学结构应用于柔性电子器件的设计与制 造。一般来说, 基于结构力学原理的剪纸拓扑结构 可以在不扩大面积和制造工艺相对简单的情况下, 赋予电子器件高拉伸性; 同时, 也可以根据实际要 求设计合适的结构来调整拉伸性能和稳定性。

宏观纸模型可以用来模拟剪纸结构的变形过 程。在弹性状态下, 变形是由结构而不是材料的尺 寸或固有机械性能决定的, 这是设计具有负泊松比 的剪纸拓扑结构的基本依据 ${ }^{[4]}$ 。2015 年, ZHANG 等 ${ }^{[45]}$ 通过优化拓扑结构或网格的附着力来提高可 拉伸性并消除应变疲劳。如图 12 所示, 通过调整拓 扑结构的尺寸参数, 最大应变可提升至 $110 \%$, 证
明了剪纸拓扑结构能够提供较大的有效拉伸应变, 同时又避免了活性材料中的明显变形，在提高柔性 电子器件的耐久性和可靠性方面有着独特的优势。
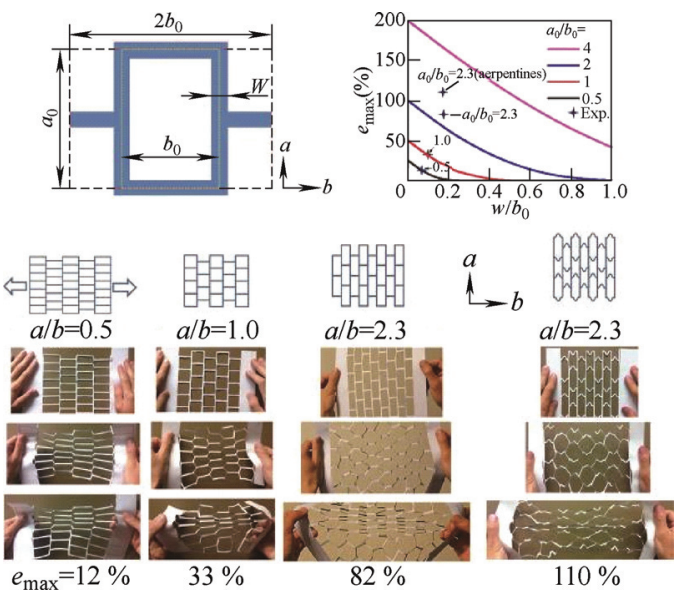

图 12 宏观纸模型模拟网络拓扑结构对拉伸性能的影响 ${ }^{[5]}$

目前, 二维的石墨烯拓扑结构通常表现出有限 的机械柔性, 其各种性能易受多维机械形变带来的 影响, 所以多尺度、多维度的剪纸拓扑结构设计成 为了近几年的研究热点。激光切割具有快速、空间 分辨率高、操作简单等优点, 是实现多尺度、多维 度拓扑结构设计的常用手段。重要的是, 激光直写 制备的石墨烯与后期拓扑结构切割加工可以相互依 存, 逐步完成。如图 13 所示, $\mathrm{LING}$ 等 ${ }^{[46]}$ 通过 $\mathrm{CO}_{2}$ 激光直写 PI 薄膜生成石墨烯并切割成二维拓扑结 构, 然后将其转移到预拉伸的硅橡胶基底上, 构建 复杂的三维石墨烯分层体系。结果表明, 这种方法 可以设计复合帐篷结构、双层螺旋结构、折纸结构、 多层体系结构等 10 多种三维分层体系, 极大地提高 了石墨烯电极的柔性化设计能力。同时, 采用有限 元分析能够精准预测这些结构的应变值, 从理论上 证明了具有剪纸拓扑结构的电子器件满足在任意维 度和多种形变条件下, 保持稳定工作能力。

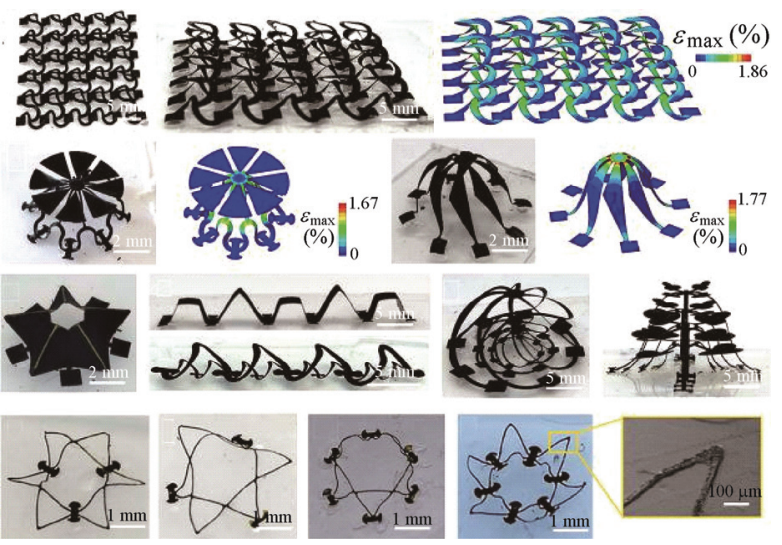

图 13 3D 剪纸拓扑结构的设计及分析 ${ }^{[46]}$ 
上述各种柔性化制造方法的相关研究成果, 不 仅为构造石墨烯基柔性电子器件提供了理论指导, 而且能够很好地与激光直写技术相结合, 引领现代 电子学的相关研究步入了柔性化发展新方向。可以 预见, 今后的研究仍会以多维度、多尺度下的拉伸 形变为重点, 推动相关理论和技术进行创新。

\section{3 应用}

随着科技的不断发展, 柔性电子器件开始走进 了人们的生活, 在健康医疗、智能家居、娱乐控制 等诸多方面扮演着重要的角色, 例如: 智能手表、 织物键盘、运动手环和心电传感器等。同时, 基于 激光直写技术制备的石墨烯基柔性电子器件也向着 多元化、集成化和多功能化方向发展。按照器件的 基本功能, 其主要研究内容可分为超级电容器、传 感器、纳米发电机和致动器四大类。

\section{1 超级电容器}

传统的供电方式主要依靠电池, 不仅续航时间 短, 而需要频繁充电或更换, 所以急需高性能的点 源设备来提供能量, 增加延长续航时间。超级电容 器, 又称电化学电容器, 具有高功率密度(比电池高 十倍)、快速充放电、长循环寿命等特点, 是柔性储 能设备发展中不可缺少的组成部分。近年来, 石墨 烯成了制造超级电容器的理想材料, 并引起了国内 外的广泛关注。利用激光直写工艺, 可以在前驱体 表面上一步构建个性化的石墨烯图案, 加以简易组 装, 即可制造与基体机械性能相似的超级电容器。

2014 年, TOUR 课题组 ${ }^{[28,47}$ 使用 $\mathrm{CO}_{2}$ 激光直写 PI 薄膜制备了具有较高电导率 $\left(2.5 \times 10^{3} \mathrm{~S} / \mathrm{m}\right)$ 的多孔 LIG 叉指电极, 并原位制作超级电容器。随后, 他们通过 PI 薄膜的激光诱导和堆叠, 制造了具有三明治结构的 超级电容器。其中, LIG 提供了较高的能量密度, 而 PI 薄膜本身的优异弯曲性能、耐温、耐酸碱能力则给 予了超级电容器在柔性电子器件中广阔的应用空间。

为了进一步改善超级电容器的能量密度和功率 密度, 2016 年, TOUR 课题组 ${ }^{[48]}$ 在 LIG 叉指电极上 沉积了多种噟电容材料, 包括二氧化锰 $\left(\mathrm{MnO}_{2}\right)$ 、羟 基氧化铁( $\mathrm{FeOOH}$ )和聚苯胺(PANI)等, 用来制备复 合超级电容器。如图 14 所示, 电极表面上的氧化还 原反应和法拉第效应相结合, 大幅度提高超级电容 器的面积电容和能量密度。其中, $\mathrm{LIG}-\mathrm{MnO}_{2}$ 电容 器的面积和体积比电容达到了 $934 \mathrm{mF} / \mathrm{cm}^{2}$ 和 93.4 $\mathrm{F} / \mathrm{cm}^{3}$, 经过 6000 次循环后, 电容保持率高达 $82 \%$, 显示出良好的循环稳定性。

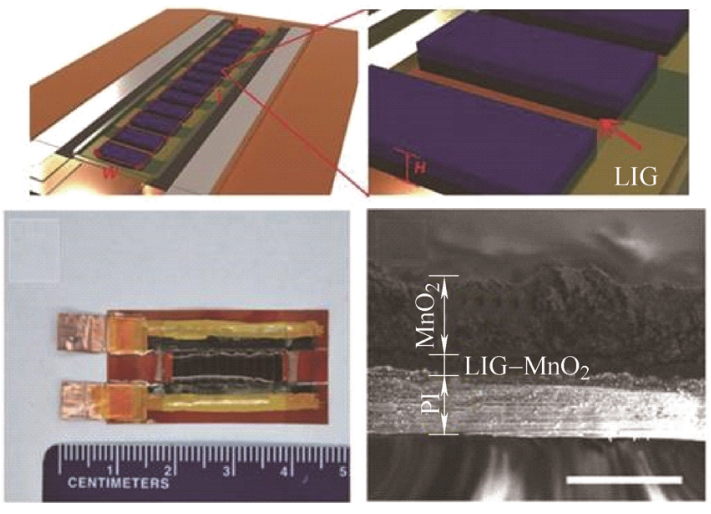

图 14 激光直写 PI 薄膜制备三明治结构的超级电容器 ${ }^{[47]}$

然而, 金属氧化物所带来的氧化还原效果毕竟 有限, 相比之下, 具有高容量、高表面积和高导电 性的过渡金属碳化物是一种更有前景的电极材料。 2019 年, 美国麻省理工学院的 ZANG 等 ${ }^{[49]}$ 以来源 于猪皮的明胶为碳源，配合 $\mathrm{MoCl}_{5} 、 \mathrm{WCl} 、 \mathrm{CoSO}_{4}$ 等多种过渡金属盐为前驱体，制备了含金属离子的 明胶溶液。随后, 将其涂抹在 PI 膜上进行激光直写, 制造掺杂超薄碳化物的 LIG, 并组装成超级电容器。 与 MXenes 和氧化物相比, 这种多晶碳化物具有分 层多孔结构, 壁厚为 $10 \sim 20 \mathrm{~nm}$, 结晶度约 $10 \mathrm{~nm}$, 显示出优异的能量存储能力。其中由 $\mathrm{MoC}_{\mathrm{x}}$ - LIG 电 容器具有较宽的耐温范围 $\left(-50 \sim 300{ }^{\circ} \mathrm{C}\right)$, 进一步提 高了 LIG 在超级电容器上的应用前景。

大尺度、多维和多样化的超级电容器同样也是 大家研究的热点。如图 15 所示, GAO 等 ${ }^{[50]}$ 通过激 光直写与切割两种工艺相结合, 开发了一种多尺度 石墨烯基超级电容器, 其不仅具有较宽的电压窗口 $(0 \sim 3 \mathrm{~V})$, 较高的面比电容 $\left(27.4 \mathrm{mF} / \mathrm{cm}^{2}\right)$ 和较高的能 量密度 $\left(32.1 \mu \mathrm{W} \cdot \mathrm{h} / \mathrm{cm}^{2}\right)$, 并且在任意方向上均显示 出优异的拉伸性能。此外, 通过模仿立体裁纸可以 制作出从一维到三维任意配置的多尺度柔性超级电 容器, 并能点亮由多个 LED 灯组成的圣诞树, 展示 出在各种柔性/可穿戴设备上的应用潜力。

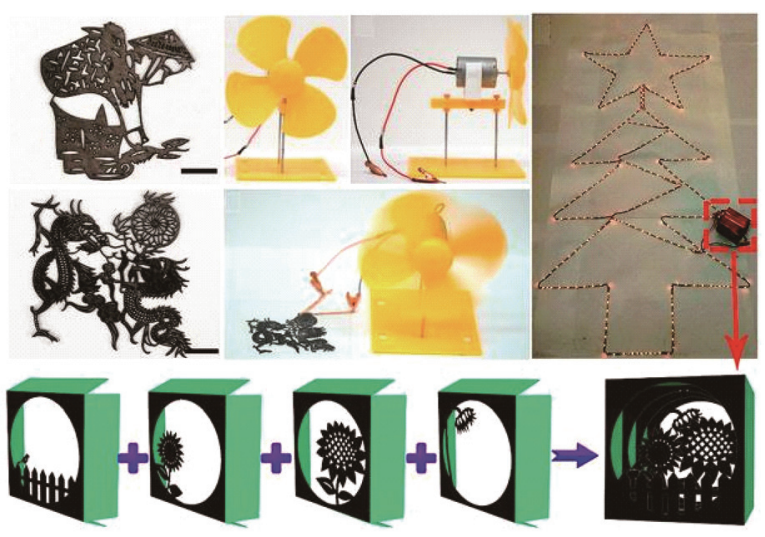

图 15 激光直写制备多尺度柔性超级电容器 ${ }^{[50]}$ 


\section{2 传感器}

传感器作为一种检测装置, 可以收集测量信息 并能将其按一定规律变换成为电信号或其他形式输 出, 以满足显示、记录和处理等功能, 是电子器件 中不可缺少的环节。通过激光直写制备石墨烯并组 装的传感器可以适用于多种测试环境, 例如: 气体、 湿度、温度、压力和应变等, 在人类健康、人机界 面、机器人等领域有着广泛的应用前景。

2014 年, TIAN 等 ${ }^{[51]}$ 利用 Light-Scribe DVD 刻 录机直写 $\mathrm{GO}$ 来制造柔性应变传感器, 通过设计石 墨烯的几何尺寸和在施加外力时石墨烯破裂所带来 的压阻效应, 应变传感器的灵敏因子从 0.11 提高到 9.49 , 具有高灵敏特点。同样, LUO 等 ${ }^{[52]}$ 也利用激 光直写工艺制备了高灵敏度石墨烯基柔性应变传感 器。柔性应变传感器可以在聚合物复合材料的结构 健康监测、手势注册和人机交互等应用中进行应变 传感/映射, 是开发定制柔性/可穿戴电子产品的有力 工具。

通过激光直写技术制备的石墨烯大多具有疏松 多孔的结构, 是一种优良的气体传感器材料, 其气 敏机制主要归因于表面上的气态分子吸附/解吸而 导致的电阻变化。基于此, STANFORD 等 ${ }^{[53]}$ 将激光 直写 PI 薄膜制造的石墨烯基气体传感器贴附在各 种材料表面上, 通过导热率所带来的电阻变化来检 测气体。另外, 石墨烯的高表面积和热导率对烟道 气中丰富的气体, 如: $\mathrm{N}_{2} 、 \mathrm{CO}$ 和 $\mathrm{CO}_{2}$ 等, 具有较 快的响应时间。所以, 该石墨烯基气体传感器可以 确定各种气体混合物的成分, 将其嵌入无机非复合 材料中, 可以制备具有电子功能的建筑材料。

目前, 大多数电子皮肤是由透气性有限的材料 制成, 限制了汗水的蒸发, 导致负面的生理/心理影 响, 不利于长期使用性。如图 16 所示, SUN 等 ${ }^{[39]}$

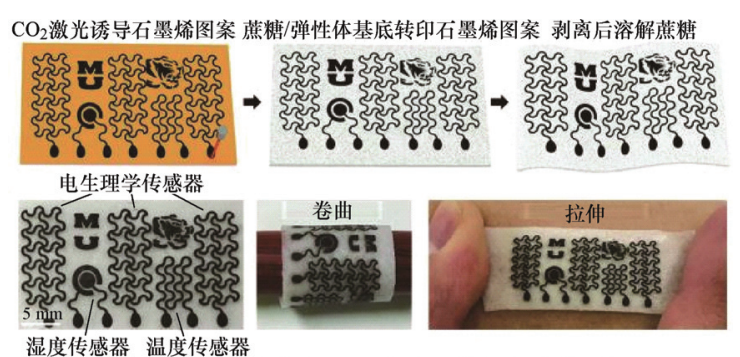

(a) 基于激光诱导LIG的可渗透多功能传感器

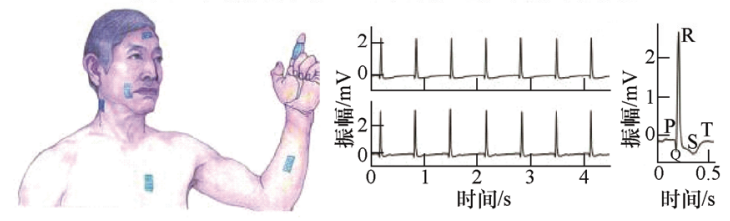

(b) 人体穿戴传感器的示意图和ECG测试

图 16 激光直写制备石墨烯基多功能海绵传感器 ${ }^{[37]}$
以激光直写 PI 薄膜生成的石墨烯作为传感元件, 硅 弹性体海绵作为祄底, 制作了多功能传感设备, 其 中包括电生理、水合和温度传感器以及焦耳发热元 件, 可以测量人体的相关生理信号, 并具有在极端 弯曲条件下给人体皮肤局部加热的功能。另外, 该 传感器的透湿率达到 $18 \mathrm{mg} \cdot \mathrm{cm}^{-2} \cdot \mathrm{h}^{-1}$, 比无孔 硅弹性体的透湿率高 18 倍, 经聚多巴胺处理后, 水 的毛细作用率高达 $1 \mathrm{~cm} / 30 \mathrm{~s}$, 与棉花相当。因此, 该多孔柔性器件可以促进排汗和蒸发, 并将皮肤炎 症风险最小化, 从而提高了人体长期穿戴的可行性。

2015 年, TIAN 等 ${ }^{[54]}$ 通过激光诱导 PI 薄膜制备 了石墨烯, 并将多个石墨烯块进行阵列化排布, 制 造了石墨烯基耳机。如图 17 所示, 在激光直写过程 中，快速气体释放导致各层之间形成气隙并膨胀， 生成了具有特殊微结构的石墨烯, 可以防止热泄漏 并提高性能。此外, 在 $100 \mathrm{~Hz}$ 至 $50 \mathrm{kHz}$ 的声音频 率上, 耳机表现出相当平坦的频率响应, 不仅对人 类(声音频率范围为 $20 \mathrm{~Hz} \sim 20 \mathrm{kHz}$ ) 有用, 而且还可 通过 $35 \mathrm{kHz}$ 声波与狗进行交流。

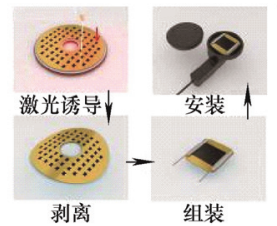

(a) 激光诱导石墨烯及组装

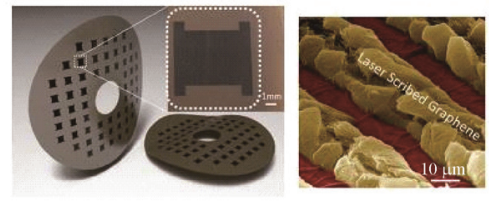

(b) LIG基传感器实物及表面形貌

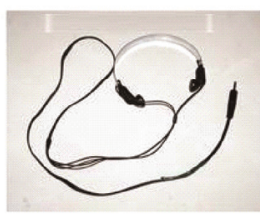

(c) 石墨烯组装的耳机

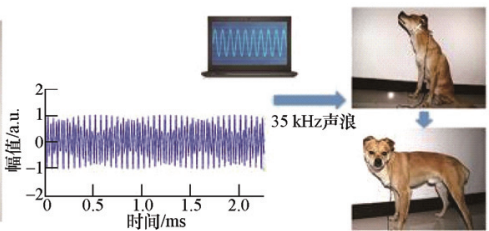

(d) 耳机通过 $35 \mathrm{kHz}$ 声音训练狗
图 17 激光直写 PI 薄膜制备石墨烯基耳机 ${ }^{[54]}$

\section{3 纳米发电机}

柔性电子器件在监测人类健康和周围环境状况 等应用中广泛使用, 但大多需要长期穿戴于人体表 面或设备, 所以减轻柔性电子器件中能量存储介质 的重量和体积, 解放人体负担成了一项重要工作, 而加入柔性能量收集器件将生活中的多余能量重新 收集存储并再次利用, 以实现可穿戴设备自供电是 一个不错的选择。

通常, 当两种材料相互接触时, 电子可以从摩 擦正电转换为摩擦负电。利用这种摩擦电效应, 纳 米发电机可以将机械能转换为电能, 并进一步存储 到储能装置中, 源源不断地为其他电子器件提供电 能。特别的是, 多种外界因素, 例如: 材料的电负 性, 组成, 环境条件和接触过程的差异等, 都会导 
致纳米发电机的发电量和效率会发生变化。

2019 年, STANFORD 等 ${ }^{[55]}$ 将采用激光直写 PI 薄膜制备石墨烯并以 PDMS 为基体进行转印, 制造 了具有高柔性和可拉伸性的纳米发电机。试验表明, 石墨烯层与多种材料介质接触均能产生电能, 其中 通过重复接触/脱离动作与皮肤的机械接触产生的 电压最高。随后, 将该纳米发电机组装在鞋底, 可 以通过人在正常行走中的脚掌与纳米发电机的接触 来发电, 最高可产生 $760 \mathrm{~V}$ 的电压。但是, 步行约 $2 \mathrm{~km}$ 后纳米发电机会变脏失效。因此, 开发一种具 有自清洁能力或者对 LIG 表面进行封装, 具有十分 重要的现实意义。
自然界中存在的大量机械能，例如: 叶片摆动、 下雨下雪、潮涨潮落等, 这些环境耗散的能量并没 有得到充分利用。为了解决这些问题并克服传统电 池的局限性, 如图 18 所示, JIANG 等 ${ }^{[56]}$ 制作了一 种高度灵活、高效的 PDMS/MXene/LIG 复合膜来组 装摩擦电纳米发电机。结果表明, 负电性的 MXene 引入到 PDMS 多孔膜中, 不仅提高了其导电性, 而 且电压输出性能显著提高。另外, 该纳米发电机可 以被应用于收集叶片摆动能量, 收集书写能量, 也 可制作成阵列用于手写识, 在农业领域、人类活动、 机器人和人机交互领域有着巨大的发展潜力。

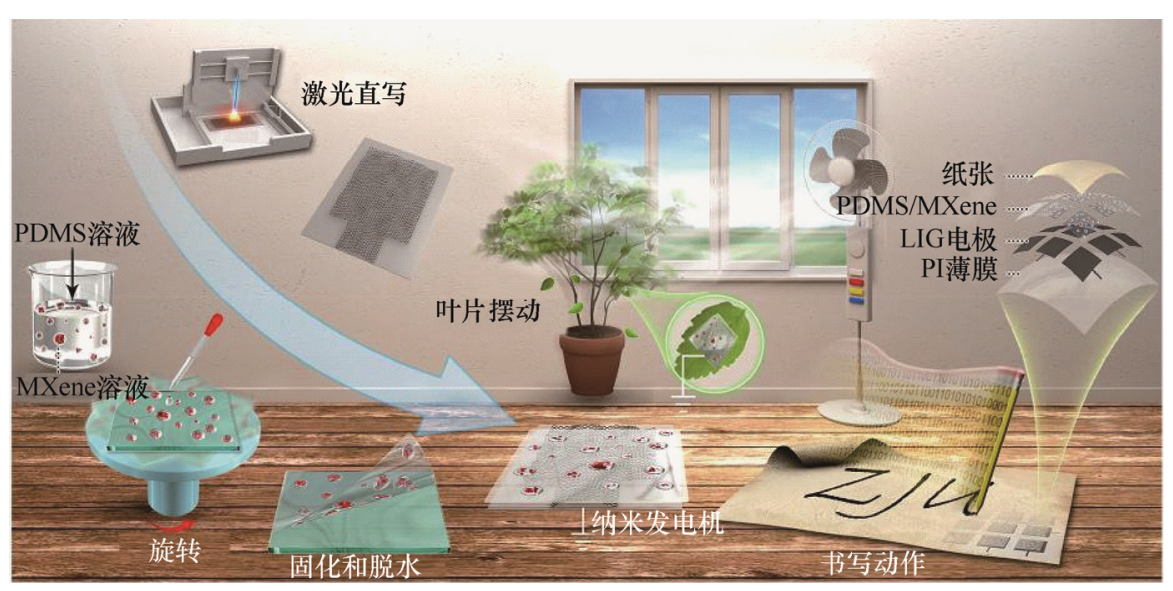

图 $18 \mathrm{PDMS} / \mathrm{MXene} / \mathrm{LIG}$ 复合膜组装纳米发电机 ${ }^{[56]}$

\section{4 致动器}

传统机械系统中的常用的致动器, 例如: 液压马 达、发动机和伺服电机等, 存在着体积庞大、精度有 限、机械结构复杂和零件磨损等问题, 已无法满足精 密器械、机器人等领域的使用需求。相反, 通过激光 直写技术制备的石墨烯是一种理想的响应式材料, 由 于其制造体系与多种平面制造技术兼容, 并且对各种 几何形状和尺寸都适用, 在发展微型化、智能化和高 精度的软体机器人和医疗设备等方面具有很大优势。

采用电流焦耳发热提供驱动力是致动器常用的 驱动方式之一。如图 19 所示, LING 等 $^{[57]}$ 通过激 光直写技术, 设计和制造了具有多种复杂三维架构 的石墨烯基电热致动器, 能够在室温下保持稳定的 三维形状结构。同时, 通过有限元方法可以定量分 析致动器的电热变化行为, 精确获得控制形状转换 的温度开关。该类致动器在机械引导的 $3 \mathrm{D}$ 组装和 人机交互领域均有良好表现, 可以参与人类手势控 制和心电图监测、人造肌肉和仿生蛙舌等应用。2014 年, $\mathrm{KIM}$ 等 ${ }^{[58]}$ 通过在 $\mathrm{GO}$ 纸的一侧进行激光直写, 制备了具有粗粘内表面和光滑外表面的非对称石墨 烯基电极。该电极具有良好的电活性, 电导率在经过
2000 次弯曲后几乎没有变化。随后, 通过石墨烯电极 与离子交换聚合物膜的组装, 制备了具有 “三明治” 结构的致动器, 其机理主要是基于固体聚电解质基质 中的离子迁移, 石墨烯具有较低的片状电阻可以减小 对致动器内电场梯度的影响, 提高器件的耐久性。

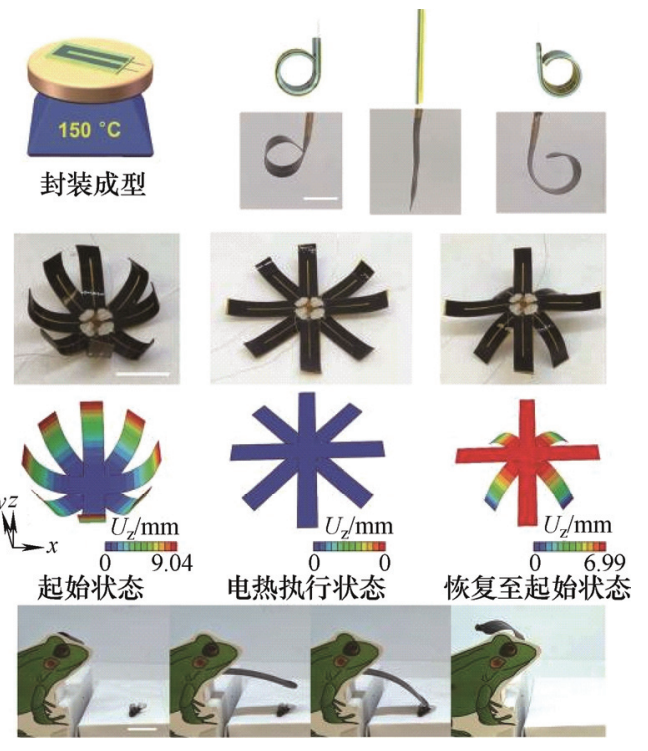

图 19 激光直写制造 LIG/PDMS 热电致动器 ${ }^{[57]}$ 
2019 年, ZHU 等 ${ }^{[59]}$ 通过激光直写技术制备了 石墨烯和聚甲基丙烯酸甲酯(PMMA)双层结构的电 热致动器, 通过调整激光扫描路径和基本工艺参数, 可以定向设计其弯曲性能, 以满足更复杂的变形控 制。研究还发现, 多种聚合物可以和石墨烯进行搭 配制造致动器, 这为激光直写制备石墨烯基柔性致 动器的研究开辟了一条新的道路。

以上研究可以发现, 激光直写制造石墨烯基柔 性电子的应用领域繁多, 产品与工艺路线等方面尚 未形成统一的标准, 产业化也是未来的阶段性目标, 但目前仍处于基础研究阶段。另外, 针对激光直写 制造方法, 建立和完善激光直写过程的理论分析模 型, 改进现有的柔性化制造工艺, 寻求可拓展、绿 色的制造模式, 并探索高效的可拓展路线是未来的 研究重点。值得注意的是, 目前石墨烯基柔性电子 器件的性能远不及日常使用要求, 理应立足于实际 应用并满足市场需求进行相关研究, 才更具有现实 意义。

\section{4 结论及展望}

目前, 传统的刚性电子器件已经不能满足市场 需求, 柔性电子器件以其可弯折、轻薄、便携等优 点逐渐成了未来的发展方向。然而, 柔性电子器件 能否大规模应用取决于原材料和制造技术。石墨烯 由自然界广泛存在的碳元素组成, 是制造柔性电子 器件的理想材料。同时, 采用激光直写技术在各种 前驱体上原位衍生石墨烯可避免高温工艺和有毒化 学物质的加入, 无需使用掩模即可实现图案化和跨 尺度结构的设计, 且与原子掺杂、金属/金属氧化复 合等多种修饰手段相容, 已在传感器、超级电容器、 纳米发电机和致动器等多种新兴器件上有所应用。另 外, 激光直写技术与卷对卷、辊对棍等自动化生产路 线兼容, 工艺简单又具有成本效益, 显示出强大的制 造潜力, 这是传统制造方法所不可比拟的。

当今, 诸多专家学者已逐渐加入到激光直写制 造石墨烯的研究行列, 美国的莱斯大学 TOUR 教授 课题组、意大利都灵理工大学的 LAMBERTI 教授课 题组、国内北京航空航天大学的罗斯达课题组和清 华大学的尤政课题组等 ${ }^{[11,19,32,37]}$ 均取得了一系列的 阶段性进展。然而, 结合激光直写石墨烯的研究现 状和应用需求, 以下几个方面需要进行重点突破。

(1) 激光直写制备石墨烯的衍生过程及其多场 因素下相互作用机制, 包括: 激光-材料间透射、折 射和反射的光场、冲击力-材料间相互作用的应力
场、光热转换和光化学反应产生的温度场、石墨烯 疏松多孔结构形成过程中释放的空气流场等, 过程 十分复杂，其相关理论未成体系。

（2）采用最优化理论和算法, 结合正交试验结 果, 构建激光直写制备石墨烯的工艺方法, 分析激 光功率、光斑大小、焦点距离和扫描速率等加工参 数和波长、频率和脉冲宽度等激光器属性对石墨烯 质量的影响; 掌握激光直写制备石墨烯的堆叠、修 饰、图案化等微纳制造工艺, 寻找高效率、高质量 的激光直写参数和最佳的加工环境。

(3) 电子器件的柔性化设计往往需要切割剪 裁、旋涂刮涂、高温脱水等外部工艺辅助设计, 缺 少独立性, 实现石墨烯的制备与柔性化制造一体化, 建立基于结构力学的剪纸拓扑结构、表面应变结构 等柔性化制造工艺方法, 可为柔性电子器件的制造 提供一个新的维度。

(4) 利用单一的激光直写技术或结合电化学沉 积、3D 打印、光刻等辅助手段, 在柔性基体上一步 制备多种石墨烯基电子元器件, 例如: 电极/电路、 传感器、晶体管和能量存储单元等, 并将其集成到 同一体系上, 以实现石墨烯基柔性电子器件的能量 采集-储存-传感-信号传输一体化设计和制造。

(5) 不同于目前成熟的无机半导体产业体系, 石墨烯基柔性电子器件离真正的应用还有很长的距 离, 许多科学问题亟需解决, 例如: 低功耗与高效 能之间的平衡设计、远程信号的传输与分析、成本 与长期使用的性能损耗、人体皮肤接触和使用安全 性等。解决多学科交叉难题是我们科研工作者面临 的挑战，同时也是发展机遇。

\section{参 考 文 献}

[1] SUN Y, ROGERS J A. Inorganic semiconductors for flexible electronics[J]. Advanced Materials, 2007, 19(15): 1897-1916.

[2] 刘长利, 沈雪石, 张学鷔, 等. 纳米电子技术的发展与 展望 $[\mathrm{J}]$. 微纳电子技术, 2011，48(10): 617-622.

LIU Changli, SHEN Xueshi, ZHANG Xueao, et al. Development and prospect of nanoelectronic technology[J]. Nanoelectronic Technology, 2011, 48(10): 617-622.

[3] HUANG Y S, LIU Y, ZHAO Y, et al. Flexible electronics: Stretchable electrodes and their future[J]. Advanced Function Materials, 2019, 29(6): 1805924.

[4] RAY T R, CHOI J G, BANDODKAR A J, et al. Bio-integrated wearable systems : A comprehensive review[J]. Chemical Reviews, 2019, 119(8): 5461-5533. 
[5] HUANG X, ZENG Z Y, Fan Z X, et al. Graphene-based electrodes[J]. Advanced Materials, 2012, 24(45) : 1897-1916.

[6] ZHAO Y, HAN Q, CHENG Z H, et al. Integrated graphene systems by laser irradiation for advanced devices[J]. Nano Today, 2017, 12: 14-30.

[7] ALLEN M J, TUNG V C, KANER R B. Honeycomb carbon: A review of graphene[J]. Chemical Reviews, 2010, 110(1): 132-145.

[8] LEWIS J A, GRATSON G M. Direct writing in three dimensions[J]. Mater. Today, 2004, 7(7): 32-39.

[9] WAN Z F, STREED E W, LOBINO M, et al. Laser-reduced graphene: Synthesis, properties, and applications[J]. Advanced Materials Technologies, 2018, 3(4): 1700315.

[10] YE R, JAMES D K, TOUR J M. Laser-induced graphene: From discovery to translation[J]. Advanced Materials, 2019, 31(1): 1803621 .

[11] YOU R, LIU Y Q, HAO Y L, et al. Laser fabrication of graphene-based flexible electronics[J]. Advanced Materials, 2019, 32(15): 1901981.

[12] DEL B E, SANCHEZ S C. Light to shape the future: From photolithography to $4 \mathrm{D}$ printing[J]. Advanced Optical Materials, 2019, 32(16): 1900598.

[13] OEHRLEIN G S, HAMAGUCHI S. Foundations of low-temperature plasma enhanced materials synthesis and etching[J]. Plasma Sources Science and Technology, 2018, 27 (2): 023001.

[14] DENG B, LIU Z F, PENG H L. Toward mass production of CVD graphene films[J]. Advanced Materials, 2019, 31(9): 1800996.

[15] BEITOLLAHI, H MOHAMMADI S Z, SAFAE M, et al. Applications of electrochemical sensors and biosensors based on modified screen-printed electrodes: A review[J]. Analytical Methods, 2020, 12(12): 1547-1560.

[16] LI Q Y, LUO S J, WANG Y, et al. Carbon based polyimide nanocomposites thin film strain sensors fabricated by ink-jet printing method[J]. Sensors and actuators a-physical, 2019, 300: 111664.

[17] KURRA N, JIANG Q, Nayak P, et al. Laser-derived graphene: A three-dimensional printed graphene electrode and its emerging applications[J]. Nano today, 2019, 24(1): 81-102.

[18] JOE D J, KIM S J, PARK J H, et al. Laser-Material Interactions for Flexible Applications[J]. Advanced
Materials, 2017, 29(26): 1606586.

[19] YE R, JAMES D K, TOUR J M. Laser-Induced Graphene[J]. Accounts of Chemical Research, 2018, 51(7): $1609-1620$.

[20] PEI S F, CHENG H M, The reduction of graphene oxide[J]. Carbon 2012, 50(9): 3210.

[21] SMIRNOV V A, ARBUZOV A A, SHULGA Y M, et al. Photoreduction of graphite oxide[J]. High Energy Chemistry Volume, 2011, 45(11): 57-61.

[22] ZHANG Y L, GUO L, WEI S, et al. Direct imprinting of microcircuits on graphene oxides film by femtosecond laser reduction[J]. Nano Today, 2010，5(1): 15-20.

[23] CHENG H, YE M, ZHAO F, et al. A general and extremely simple remote approach toward graphene bulks with in stimuli functionalization[J]. Advanced Materials, 2016, 28(17): 3305-3312.

[24] GUO L, ZHANG Y L, HAN D D, et al. Laser-mediated programmable $\mathrm{N}$-doping and simultaneous reduction of graphene oxides[J]. Advanced Optical Materials, 2013, 2 (2): 120 .

[25] ABDELSAYED V, MOUSSA S, HASSAN H M, et al. Photothermal deoxygenation of graphite oxide with laser excitation in solution and graphene-aided increase in water temperature $[\mathrm{J}]$. The Journal of Physical Chemistry Letters, 2010, 1(19): 2804-2809.

[26] HUANG L, LIU Y, JI L C, et al. Pulsed laser assisted reduction of graphene oxide[J]. Carbon, 2011, 49(7): 2431.

[27] SRINIVASAN R, HALL R R, WILSON W D, et al. Formation of a porous, patternable, electrically conducting carbon network by the ultraviolet laser irradiation of the polyimide PDMDA-ODA (Kapton) [J]. Chemistry of Materials, 1994, 6(7): 888-889.

[28] LIN J, PENG Z, LIU Y, et al. Laser-induced porous graphene films from commercial polymers[J]. Nature Communications, 2014, 5: 5714

[29] ZHANG Z C, SONG M M, HAO J X, et al. Visible light laser-induced graphene from phenolic resin: A new approach for directly writing graphene-based electrochemical devices on various substrates[J]. Carbon, 2018, 127: 278-296

[30] LI Y L, LUONG X X, ZHANG J B, et al. Laser-induced graphene in controlled atmospheres : From superhydrophilic to superhydrophobic surfaces[J]. Advanced Function Materials, 2017， 29(27): 1700496. 
[31] LUONG D X, SUBRAMANIAN A K, SILVA G A L, et al. Laminated object manufacturing of 3D-printed laser-induced graphene foams[J]. Advanced Materials, 2018, 30(28): 1707416.

[32] WANG Y N, WANG Y, ZHANG P P, et al. Laser-induced freestanding graphene papers: A new route of scalable fabrication with tunable morphologies and properties for multifunctional devices and structures[J]. Small, 2018, $14(36): 1802350$.

[33] YE R, CHYAN Y, ZHANG J, et al. Laser-induced graphene formation on $\operatorname{wood}[\mathrm{J}]$. Advanced Materials, 2017, 29(37): 1702211.

[34] CHYAN Y, YE R, LI Y, et al. Laser-induced graphene by multiple lasing: Toward electronics on cloth, paper, and food[J]. ACS Nano， 2018， 12(3): 2176-2183.

[35] LE T S D, PARK S, AN J N, et al. Ultrafast laser pulses enable one - step graphene patterning on woods and leaves for green electronics[J]. Advanced Function Materials, 2019, 29(33): 1902771.

[36] ZANG X N, JIAN C, INGERSOLL S, et al. Laser-engineered heavy hydrocarbons: Old materials with new opportunities[J]. Science Advances, 2020，6: 5231.

[37] LAMBERTI A, CLERICI F, FONTANA $M$, et al. Highly stretchable supercapacitor using laser-induced graphene electrodes onto elastomeric substrate[J]. Advanced Energy Materials, 2016, 6(10): 1600050.

[38] SONG W, ZHU J, GAN B, S. et al. Flexible, stretchable, and transparent planar microsupercapacitors based on 3D porous laser-induced graphene[J]. Small, 2018, 14(1): 1702249.

[39] WANG W T, LU L S, XIE Y X, et al, A highly stretchable microsupercapacitor using laser-induced graphene/NiO/ $\mathrm{Co}_{3} \mathrm{O}_{4}$ electrodes on a biodegradable waterborne polyurethane substrate[J]. Advanced Materials Technology, 2020，5(2): 1900903.

[40] SUN B H, MCCAY R N, GOSWAMI S, et al. Gas-permeable, multifunctional on-skin electronics based on laser-induced porous graphene and sugar-templated elastomer sponges[J]. Advanced Materials, 2018, 30(50): 1804327.

[41] OH J S, KIM S H, HWANG T, et al. Laser-assisted simultaneous patterning and transferring of graphene[J]. The Journal of Physical Chemistry C, 2013, 117(1): 663-668.

[42] KIM K, PARK Y G, HYUN B G, et al. Recent advances in transparent electronics with stretchable forms[J]. Advanced Materials, 2018，31(20): 1804690.

[43] QI D P, LIU Z Y, LIU Y, et al. Suspended wavy graphene microribbons for highly stretchable microsupercapacitors[J]. Advanced Materials， 2015， 27(37): 5559-5566.

[44] GUO C F, LIU Q, WANG G, et al. Fatigue-free, superstretchable, transparent, and biocompatible metal electrodes[J]. PNAS, 2015, 112(40): 12332.

[45] ZHANG Y H, YAN Z, NAN K, et al. A mechanically driven form of Kirigami as a route to $3 \mathrm{D}$ mesostructures in micro/nanomembranes[J]. PANS, 2015, 112(38): 11757.

[46] LING Y, ZHUANG X T, XU Z, et al. Mechanically assembled, three-dimensional hierarchical structures of cellular graphene with programmed geometries and outstanding electromechanical properties[J]. ACS Nano, 2018, 12(12): 12456-12463.

[47] PENG Z W, LI J, YE R, et al. Flexible and stackable laser-induced graphene supercapacitors[J]. ACS Applied Materials \& Interfaces, 2015, 7(5): 3414-3419.

[48] LI L, ZHANG J B, PENG Z W, et al. High-performance pseudocapacitive microsupercapacitors from laser-induced graphene[J]. Advanced Materials, 2016, 28(5): 838-845

[49] ZANG X N, JIAN C Y, ZHU T S, et al. Laser-sculptured ultrathin transition metal carbide layers for energy storage and energy harvesting applications[J]. Nature Communication, 2019, 10: 1-8.

[50] GAO J, SHAO C X, SHAO S X, et al. Laser-assisted multiscale fabrication of configuration-editable supercapacitors with high energy density[J]. ACS Nano, 2019, 13(7): 7463-7470.

[51] TIAN H, SHU Y, CUI Y L, et al. Scalable fabrication of high-performance and flexible graphene strain sensors[J]. Nanoscale, 2014, 6(2): 699.

[52] LUO S, HOANG P T, LIU T, et al. Direct laser writing for creating porous graphitic structures and their use for flexible and highly sensitive sensor and sensor arrays[J]. Carbon, 2016, 96: 522.

[53] STANFORD M G, YANG K C, CHYAN Y, et al. Laser-induced graphene for flexible and embeddable gas sensors[J]. ACS Nano, 2019, 13(3): 3474-3482.

[54] TIAN H, SHU Y, WANG X F, et al. A graphene-based resistive pressure sensor with record-high sensitivity in a wide pressure range[J]. Scientific Reports, 2015, 5: 8603 
[55] STANFORD M G, LI J T, CHYAN Y, et al. Laser-induced graphene triboelectric nanogenerators[J]. ACS Nano 2019, 13(6): 7166-7174

[56] JIANG C M, Li X J, YAO Y, et al. A multifunctional and highly flexible triboelectric nanogenerator based on MXene-enabled porous film integrated with laser-induced graphene electrode[J]. Nano Energy, 2019, 66: 104121.

[57] LING Y, PANG W B, LI X P, et al. Laser-induced graphene for electrothermally controlled, mechanically guided, 3D assembly and human - soft actuators interaction[J]. Advanced Materials, 2020: 1908475.

[58] KIM J W, JEON J H, KIM H J, et al. Durable and water-floatable ionic polymer actuator with hydrophobic and asymmetrically laser-scribed reduced graphene oxide paper electrodes[J]. ACS Nano，2014， 8(3): 2986-2997

[59] ZHU L, GAO Y Y, HAN B, et al. Laser fabrication of graphene-based electrothermal actuators enabling predicable deformation[J]. Optics Letters, 2019, 44(6):

$1363-1366$

作者简介: 陆龙生, 男, 1981 年出生, 博士, 教授, 博士研究生导师。 主要研究方向为表面微纳功能结构制造。

E-mail: meluls@scut.edu.cn

王文涛, 男, 1993 年出生, 博士研究生。主要研究方向为激光加工和柔 性电子器件制造。

E-mail: wangwentao@scut.edu.cn

谢颖熙(通信作者), 男, 1989 年出生, 博士, 副教授。主要研究方向为 碳基材料微纳米加工、激光加工和微超级电容器。

E-mail: xieyingxi@scut.edu.cn

汤勇, 男, 1962 年出生, 博士, 教授, 博士研究生导师。主要研究方向 为微制造、表面功能结构制造。

E-mail: ytang@scut.edu.cn 